\title{
The 16th International Symposium on Relations Between Homogeneous and Heterogeneous Catalysis (ISHHC-16), Sapporo, August 4-9, 2013
}

\author{
Yasuhiro Iwasawa $\cdot$ Atsushi Fukuoka $\cdot$ \\ Kiyotaka Asakura $\cdot$ Hiromi Yamashita
}

Published online: 4 March 2014

(c) Springer Science+Business Media New York 2014

This is the special issue containing 34 articles based on papers presented at the 16th International Symposium on Relations between Homogeneous and Heterogeneous Catalysis (ISHHC-16), held at Hokkaido University, Sapporo, Japan, 4-9 August 2013. The aim of this symposium was to bring together researchers in the three fields of heterogeneous, homogeneous and enzymatic catalyses, and to provide all participants with opportunities to have more profound understanding and integration of fundamental concepts and latest developments of the three fields. This is one of the series of the ISHHCs which were held in Brussels (1974), Lyon (1977), Groningen (1981), Asilomar (1983), Novosibirsk (1986), Pisa (1989), Tokyo (1992), Balatonfured (1995), Southampton (1999), Lyon (2001), Evanston (2003), Florence (2005), Berkeley (2007), Stockholm (2009) and Berlin (2011).

Catalysis is the key to creating a sustainable society, and for that purpose we need to reinforce catalytic science and technology and to extend our findings to innovation. Catalysis is now not only a mere research category of natural science but also has become a common word used figuratively regarding essential relationships in various kinds of human activity including science, economy, and politics. At this symposium, papers on the following topics were welcome: (1) New Concepts and Mechanisms of Catalysis, (2) New Methods and Characterization in Catalysis, (3)

\footnotetext{
Y. Iwasawa $(\bowtie)$

The University of Electro-Communications, Tokyo, Japan

e-mail: iwasawa@pc.uec.ac.jp

A. Fukuoka $\cdot$ K. Asakura

Hokkaido University, Sapporo, Japan

H. Yamashita

Osaka University, Osaka, Japan
}

Advanced Materials for Catalysis, (4) Catalysis for Green and Sustainable Chemistry, (5) Catalysis for Fine Chemicals, (6) Bio-inspired Catalysts, (7) Energy and Environmental Catalysts, and (8) New Industrial Catalysts and Processes.

Japan has been undergoing reconstruction since the Japanese Great Earthquake and Tsunami of 2011. Since then, we have realized again the close bonds among people in Japan, as well as between the people of Japan and elsewhere in the world. Therefore, we have adopted the Japanese word Kizuna (meaning "bond") for the logo of the ISHHC-16 to represent the close bond knitting together our community.

In the ISHHC-16, we had 7 plenary lectures, 17 keynote lectures, 96 oral presentations and 230 posters, among which 13 young scientists received Poster Awards. We also had three evening sessions devoted to: (i) the R\&D challenges facing Japanese chemical companies, (ii) Catalysis Research Center and ISHHC, and (iii) molecular activation. These sessions were very useful in revealing the present situation and future prospects in academia and industry related to catalysis.

We were pleased to welcome at ISHHC 503 participants from 30 countries, which reflected the international dimension of the symposium. We hope that the presentations and discussion in the ISHHC-16 will contribute to mutual understanding in our community and further development of catalytic science and technology. It was also recognized at ISHHC-16 that education for young scientists and students to understand the fundamentals of homogenous, heterogeneous and enzymatic catalysis is important for the concerted development of these research fields.

We are grateful to all our sponsors who have provided generous supports for the symposium. We also thank the international advisory board and organizing committee members.

The next ISHHC-17 will take place in Utrecht, The Netherlands in July 2015. 